ANNALES

POLONICI MATHEMATICI

$80(2003)$

\title{
Intersection of analytic curves
}

\author{
by TADEusz KRAsiński (Łódź) and \\ KRzyszTOF JAN NowAK (Kraków)
}

Dedicated to Professor Józef Siciak
on the occasion of his 70th birthday

\begin{abstract}
We give a relation between two theories of improper intersections, of Tworzewski and of Stückrad-Vogel, for the case of algebraic curves. Given two arbitrary quasiprojective curves $V_{1}, V_{2}$, the intersection cycle $V_{1} \bullet V_{2}$ in the sense of Tworzewski turns out to be the rational part of the Vogel cycle $v\left(V_{1}, V_{2}\right)$. We also give short proofs of two known effective formulae for the intersection cycle $V_{1} \bullet V_{2}$ in terms of local parametrizations of the curves.
\end{abstract}

1. Introduction. For two arbitrary purely dimensional analytic subsets $V_{1}, V_{2}$ (or, in general, for two analytic cycles $V_{1}, V_{2}$ ) of a complex manifold $M$, Tworzewski [T] defined an intersection product $V_{1} \bullet V_{2}$ which is an analytic cycle. This theory was initiated in the case of improper isolated intersections by Achilles, Winiarski and Tworzewski [ATW], who made use of Draper's ideas (cf. [D]) concerning proper intersections in complex analytic geometry. The intersection cycle $V_{1} \bullet V_{2}$ coincides with the classical one for every proper intersection of $V_{1}$ and $V_{2}$. On the other hand, for two arbitrary purely dimensional quasiprojective varieties $V_{1}, V_{2}$ over a field $K$ we have the Vogel intersection cycle $v\left(V_{1}, V_{2}\right)$ (see [CFV, G1, G2]), which is an algebraic cycle defined over a pure transcendental extension of $K$. So, we may distinguish in $v\left(V_{1}, V_{2}\right)$ its rational part $v_{\text {rat }}\left(V_{1}, V_{2}\right)$ (i.e. the part defined over $K)$ and transcendental part $v_{\text {tr }}\left(V_{1}, V_{2}\right)$ :

$$
v\left(V_{1}, V_{2}\right)=v_{\text {rat }}\left(V_{1}, V_{2}\right)+v_{\text {tr }}\left(V_{1}, V_{2}\right) .
$$

2000 Mathematics Subject Classification: 14C17, 32B15.

Key words and phrases: improper intersection, analytic and algebraic curves, Tworzewski intersection cycle, Vogel intersection cycle, normal cone, relative tangent cone.

The authors were partially supported by KBN Grants: No. 2 P03A 00718 (the first) and No. 5 P03A 00521 (the second). 
The main result of the paper (Theorem 1) asserts that if $V_{1}, V_{2}$ are arbitrary quasiprojective curves over $\mathbb{C}$, then

$$
V_{1} \bullet V_{2}=v_{\text {rat }}\left(V_{1}, V_{2}\right) \text {. }
$$

For plane algebraic curves, this was obtained by the first author in [K2] by other methods. The proof of the main theorem is based on the method of deformation to the normal cone for analytic improper intersections, investigated by the second author in [N1, N2, N3]. Namely, we analyze the normal cone

$$
C:=C_{\left(V_{1} \times V_{2}\right) \cap \Delta}\left(V_{1} \times V_{2}\right)
$$

to $\left(V_{1} \times V_{2}\right) \cap \Delta$ in $V_{1} \times V_{2}$, where $\Delta$ is the diagonal.

The other results are new short proofs of two known effective formulae for the intersection cycle $V_{1} \bullet V_{2}$ of analytic curves $V_{1}$ and $V_{2}$ in terms of local parametrizations of $V_{1}$ and $V_{2}$ (Theorem 2). Whereas the first formuladue to Chądzyński, Krasiński and Tworzewski [CKT]—refers to an isolated intersection, the second one - due to the first author [K2] - is concerned with self-intersection. What makes it possible to simplify the reasonings is a theorem by the second author [N4] to the effect that the generalized intersection index $\widetilde{g}$ is realized by every collection of hyperplanes admissible with respect to the tangent cone $B$ at $P$ to the support of the normal cone $C$.

2. Normal cones. For two arbitrary purely dimensional analytic subsets $V_{1}, V_{2}$ of a complex manifold $M$, Tworzewski [T] defined, by means of the analytic intersection algorithm, a generalized intersection index $\tilde{g}$ of $V_{1}$ and $V_{2}$ at a point $P$ (which is a sequence of non-negative integers), an intersection index $g$ (the sum of the components of $\tilde{g}$ ) and an intersection product $V_{1} \bullet V_{2}$ (an analytic cycle such that $\operatorname{mult}_{P}\left(V_{1} \bullet V_{2}\right)=g(P)$ for $\left.P \in M\right)$. The intersection product $V_{1} \bullet V_{2}$ coincides with the classical one for every proper analytic intersection. The intersection index $g$ at a point $P$ for an analytic set $V$ and a submanifold $S$ of the ambient manifold $M$ coincides with the multiplicity at $P$ of the normal cone $C:=C_{V \cap S} V$ (cf. [AR, N1, N2, N3]), where the intersection $V \cap S$ is understood in the ideal-theoretic sense, i.e. as a possibly non-reduced analytic subspace of $M$. One may regard the normal cone $C$ both as an analytic space and an analytic cycle. Throughout the paper we adopt the latter meaning.

A diagonal procedure reduces the study of intersections of two analytic subsets $V_{1}$ and $V_{2}$ of a complex manifold $M$ to that of intersections of the product $V_{1} \times V_{2}$ with the diagonal $\Delta$ which is a submanifold of the product $M \times M$. Therefore, the index $g$ of intersection at $P$ of two analytic sets $V_{1}$ and $V_{2}$ is the multiplicity at $(P, P)$ of the normal cone

$$
C:=C_{\left(V_{1} \times V_{2}\right) \cap \Delta}\left(V_{1} \times V_{2}\right) .
$$


We now recall a geometric construction of $C$ (see e.g. [N3, Ch. II, Sects. 3 and 4]). Define the following family of analytic sets parametrized by $\lambda \in$ $\mathbb{C} \backslash\{0\}:$

$\left\{(x, y, \lambda(x-y) ; 1: \lambda): x \in V_{1}, y \in V_{2}, \lambda \in \mathbb{C}, \lambda \neq 0\right\} \subset \mathbb{C}_{x}^{m} \times \mathbb{C}_{y}^{m} \times \mathbb{C}_{v}^{m} \times \mathbb{P}_{1}$. The closure $\mathcal{V}$ of this family in $\mathbb{C}_{x}^{m} \times \mathbb{C}_{y}^{m} \times \mathbb{C}_{v}^{m} \times \mathbb{P}_{1}$ is an analytic set, and the fibre $\mathcal{V}_{\infty}$ of $\mathcal{V}$ over $\lambda=\infty$ (which may be regarded both as an analytic subspace and an analytic cycle) is the normal cone $C$. The normal cone $C=\mathcal{V}_{\infty}$ is the limit of the cycles $\mathcal{V}_{\lambda}$ (the fibres of $\mathcal{V}$ over $\lambda$ ) in the topology of locally uniform convergence of positive cycles. From the set-theoretical viewpoint, the support of the cone $C$ is the analytic set

$$
\begin{array}{r}
|C|=\left\{(x, x, v) \in V_{1} \times V_{2} \times \mathbb{C}_{v}^{m}: x_{n}, y_{n} \rightarrow x \text { for some } x_{n} \in V_{1}, y_{n} \in V_{2},\right. \\
\text { and } \left.\lambda_{n}\left(x_{n}-y_{n}\right) \rightarrow v \text { for some } \lambda_{n} \rightarrow \infty\right\} .
\end{array}
$$

From now on we shall consider two analytic curves $V_{1}$ and $V_{2}$. Then the normal cone $C$ is an analytic cone of pure dimension 2. Since the intersection problem in question is local, we may view $V_{1}, V_{2}$ as germs at a fixed point $P=0 \in \mathbb{C}^{m}$.

The constructions of normal cones and intersection indices are additive (see e.g. [N3, Ch. II, Sect. 3]). Consequently, if $V_{1 i}(i=1, \ldots, k)$ and $V_{2 j}(j=$ $1, \ldots, l)$ are the irreducible components of the germs $V_{1}$ and $V_{2}$ respectively, the (generalized) intersection index for the pair $V_{1}, V_{2}$ is the sum of those for the $k l$ pairs $V_{1 i}, V_{2 j}$, and

$$
C=\sum_{i, j} C_{\left(V_{1 i} \times V_{2 j}\right) \cap \Delta}\left(V_{1 i} \times V_{2 j}\right) .
$$

One may thus confine oneself to the case where the analytic curve germs $V_{1}$ and $V_{2}$ are irreducible. There are two different cases:

- $V_{1} \neq V_{2}$, and then $C=C_{0}$ is an algebraic cone with vertex at the origin;

- $V_{1}=V_{2}=V$, and then $C$ is the sum of an analytic cone $C^{\prime}$ over $V^{\Delta}:=(V \times V) \cap \Delta$ (counted with coefficient 1$)$ and an algebraic cone $C_{0}$ with vertex at the origin.

For the case of self-intersection we show that the multiplicity at the origin of the analytic cone $C^{\prime}$ is equal to the multiplicity at $P=0$ of the curve $V$ (Proposition 1). From this we will deduce the main theorem of the paper (Theorem 1).

We should mention that - in view of the geometric description (1) of the normal cone $C$ (see also [N3, Ch. II, Sects. 3 and 4]) - for arbitrary analytic sets $V_{1}$ and $V_{2}$, the support of the fibre over $(P, P)$ of the cone $C$ is the relative tangent cone to $V_{1}$ and $V_{2}$ at $P$ (defined in [ATW]). The relative tangent cones to analytic curves were studied by Ciesielska $[\mathrm{C}]$ and the first 
author [K1]; in particular, the paper [K1] describes the relative tangent cone in terms of parametrizations of the curves.

Proposition 1. Suppose $V$ is an irreducible curve germ at $P=0 \in \mathbb{C}^{m}$ and $C:=C_{V} \Delta(V \times V)$. Let $C^{\prime}$ be the part of the normal cone $C$ that lies over $V^{\Delta}$. Then the multiplicity of $C^{\prime}$ at the origin coincides with the multiplicity of $V$ at $P=0$.

Proof. Indeed, $V$ can be parametrized, in suitable coordinates near the origin, as follows:

$$
x_{1}=t^{p}, \quad x_{2}=\varphi_{2}(t), \ldots, x_{m}=\varphi_{m}(t)
$$

with $\operatorname{ord}_{0} \varphi_{k}>p$. Obviously, the multiplicity of $V$ at $P=0$ is $p$ and the tangent line to $V$ at $P$ is $\left\{(t, 0, \ldots, 0) \in \mathbb{C}^{m}: t \in \mathbb{C}\right\}$. Put $\varphi(t)=$ $\left(\varphi_{2}(t), \ldots, \varphi_{m}(t)\right)$; then it is easy to check that

$$
\left(t^{p}, \varphi(t), t^{p}, \varphi(t), p s t^{p-1}, s \varphi^{\prime}(t)\right)
$$

is a parametrization of the cone $C^{\prime}$. Since

$$
\operatorname{ord}_{(0,0)}\left(t^{p}, \varphi(t), t^{p}, \varphi(t), p s t^{p-1}, s \varphi^{\prime}(t)\right)=p,
$$

it follows that $\operatorname{mult}_{P} C^{\prime}=p$, as desired.

3. The main result. We apply Proposition 1 to show that the intersection cycle $V_{1} \bullet V_{2}$ is the rational part of the Vogel cycle $v\left(V_{1}, V_{2}\right)$.

THEOREM 1. For any quasiprojective curves $V_{1}$ and $V_{2}$, the intersection cycle $V_{1} \bullet V_{2}$ is the rational part $v_{\text {rat }}\left(V_{1}, V_{2}\right)$ of the Vogel cycle $v\left(V_{1}, V_{2}\right)$.

Proof. Since the contructions of the intersection cycle and Vogel cycle are local, we can confine ourselves to the affine case. By additivity, we may assume that the curves $V_{1}, V_{2}$ are irreducible. There are two cases: $V_{1} \neq V_{2}$ or $V_{1}=V_{2}=V$. We keep the foregoing notation: the normal cone $C$ is the sum of the cones $C_{0}$ and $C^{\prime}$. While in the first case $C^{\prime}=0$ and $C_{0}$ is a finite sum of algebraic cones with vertices at $(P, P), P \in V_{1} \cap V_{2}$, in the second $C^{\prime}$ is a cone over $V^{\Delta}$ (counted with coefficient 1 ) and $C_{0}$ is a finite sum of algebraic cones with vertices at $(P, P), P \in$ singular locus of $V$.

In the first case both $V_{1} \bullet V_{2}$ and $v\left(V_{1}, V_{2}\right)=v_{\text {rat }}\left(V_{1}, V_{2}\right)$ are 0 -cycles on $V_{1} \cap V_{2}$ :

$$
V_{1} \bullet V_{2}=\sum i\left(V_{1} \bullet V_{2} ; P\right) \cdot[P], \quad v_{\text {rat }}\left(V_{1}, V_{2}\right)=\sum \varepsilon_{P} \cdot[P],
$$

where $P$ ranges over $V_{1} \cap V_{2}$. But both the coefficients $\varepsilon_{P}$ and $i\left(V_{1} \bullet V_{2} ; P\right)$ are equal to the multiplicity at the vertex $(P, P)$ of the normal cone $C$; these equalities follow from the fact that the Vogel cycle is invariant under deformation to the normal cone (cf. [G2]) and from the description of the intersection index by normal cones (cf. [AR, N1, N2, N3]). Hence $V_{1} \bullet V_{2}=$ $v_{\text {rat }}\left(V_{1}, V_{2}\right)$, as desired. 
In the case of self-intersection,

$$
\begin{aligned}
V \bullet V & =[V]+\sum i(V \bullet V ; P) \cdot[P], \\
v_{\text {rat }}(V, V) & =[V]+\sum \varepsilon_{P} \cdot[P],
\end{aligned}
$$

where $P$ ranges over the singular locus of $V$. Again, as before, $\varepsilon_{P}$ is equal to the multiplicity at $(P, P)$ of the algebraic cone $C_{0}$ because the Vogel cycle is invariant under deformation to the normal cone (cf. [G2] and also [G1, Chap. 3, Sect. 2A]). On the other hand, we have

$$
\operatorname{mult}_{P}(V \bullet V)=\text { mult }_{P} V+i(V \bullet V ; P),
$$

and $\operatorname{mult}_{P}(V \bullet V)$ is, by definition, equal to the intersection index $g(P)$, which again coincides with the multiplicity of the normal cone $C$. Therefore

$$
\operatorname{mult}_{P} V+i(V \bullet V ; P)=\operatorname{mult}_{(P, P)} C=\operatorname{mult}_{(P, P)} C^{\prime}+\operatorname{mult}_{(P, P)} C_{0} .
$$

Hence and by Proposition 1, we obtain

$$
i(V \bullet V ; P)=\operatorname{mult}_{(P, P)} C_{0}=\varepsilon_{P}
$$

which completes the proof.

EXAmple 1. Consider the self-intersection of the curve

$$
V:=\left\{(x, y): y^{2}-x^{3}=0\right\}
$$

in $\mathbb{C}^{2}$ (for simplicity we do not consider this intersection in $\mathbb{P}^{2}$ ). Using the intersection algorithms of both theories we obtain

$$
\begin{gathered}
V \bullet V=1 \cdot[V]+3 \cdot[(0,0)] \quad(\text { a cycle over the field } \mathbb{C}), \\
v(V, V)=1 \cdot[V]+3 \cdot[(0,0)]+1 \cdot\left[\left(\frac{4 a^{2}}{9 b^{2}},-\frac{8 a^{3}}{27 b^{3}}\right)\right]
\end{gathered}
$$

(a cycle over the field $\mathbb{C}(a, b))$.

The construction of the Vogel cycle imposes a lower bound on the dimensions of its components. Since no such bound exists for intersection cycles, the cycles $V_{1} \bullet V_{2}$ and $v_{\text {rat }}\left(V_{1}, V_{2}\right)$ need not coincide for higher dimensional sets $V_{1}, V_{2}$. We illustrate this by Example 2 (Ex. 5 from [N3, Ch. III, Sect. 3]) concerning the self-intersection of a projective surface. We should also emphasize that while the Vogel cycle $v\left(V_{1}, V_{2}\right)$ is effective, $V_{1} \bullet V_{2}$ may not be an effective cycle, as demonstrated in Example 3.

EXAMPLE 2. Let $V$ be the cubic surface in $\mathbb{P}^{3}$ given by the equation

$$
z_{0} z_{1} z_{2}+z_{1} z_{2} z_{3}+z_{2} z_{3} z_{0}+z_{3} z_{0} z_{1}=0 .
$$

Its singular locus consists of only four nodes (i.e. non-degenerate isolated singular points of degree 2$)$

$$
\begin{array}{ll}
P_{0}=(1: 0: 0: 0), & P_{1}=(0: 1: 0: 0), \\
P_{2}=(0: 0: 1: 0), & P_{3}=(0: 0: 0: 1) .
\end{array}
$$


We have (cf. [N3, Ch. III, Sect. 3, Ex. 5])

$$
V \bullet V=1 \cdot[V]+2 \cdot\left[P_{0}\right]+2 \cdot\left[P_{1}\right]+2 \cdot\left[P_{2}\right]+2 \cdot\left[P_{3}\right] .
$$

On the other hand, the Vogel cycle $v\left(V_{1}, V_{2}\right)$ is the sum of $[V]$ and a transcendental curve of degree 6 through $P_{0}, P_{1}, P_{2}, P_{3}$. Hence

$$
v_{\text {rat }}\left(V_{1}, V_{2}\right)=1 \cdot[V] \neq V \bullet V .
$$

Example 3. Consider two affine sets in $\mathbb{C}^{6}=\mathbb{C}_{\mathbf{x}}^{4} \times \mathbb{C}_{y, z}^{2}, \mathbf{x}=\left(x_{1}, x_{2}\right.$, $\left.x_{3}, x_{4}\right)$, defined by

$$
V_{1}:=\left\{(\mathbf{x}, y, z): z x_{1} x_{2}=y x_{3} x_{4}\right\}, \quad V_{2}:=\{(\mathbf{x}, y, z): z=y=0\} .
$$

By straightforward calculations we obtain

$$
\begin{aligned}
V_{1} \bullet V_{2}= & 1 \cdot\left[V_{2}\right]+1 \cdot\left[\mathbb{C}_{x_{1}, x_{3}}^{2}\right]+1 \cdot\left[\mathbb{C}_{x_{1}, x_{4}}^{2}\right]+1 \cdot\left[\mathbb{C}_{x_{2}, x_{3}}^{2}\right]+1 \cdot\left[\mathbb{C}_{x_{2}, x_{4}}^{2}\right] \\
& -1 \cdot\left[\mathbb{C}_{x_{1}}\right]-1 \cdot\left[\mathbb{C}_{x_{2}}\right]-1 \cdot\left[\mathbb{C}_{x_{3}}\right]-1 \cdot\left[\mathbb{C}_{x_{4}}\right]+2 \cdot[0], \\
v\left(V_{1}, V_{2}\right)= & 1 \cdot\left[V_{2}\right]+v^{2},
\end{aligned}
$$

where $v^{2}$ is the cycle of dimension 3 on $V_{2} \otimes_{\mathbb{C}} \mathbb{C}(a, b)=\mathbb{C}(a, b)^{4}$ determined by the equation

$$
a x_{1} x_{2}+b x_{3} x_{4}=0
$$

Hence

$$
v_{\text {rat }}\left(V_{1}, V_{2}\right)=1 \cdot\left[V_{2}\right] \neq V_{1} \bullet V_{2} .
$$

4. Formulae for intersection cycles. For two analytic curve germs $V_{1}, V_{2}$ at $P=0 \in \mathbb{C}^{m}$, put

$$
C:=C_{\left(V_{1} \times V_{2}\right) \cap \Delta}\left(V_{1} \times V_{2}\right),
$$

and let $C^{\prime}$ and $C_{0}$ be the parts of $C$ that lie over $\left(V_{1} \times V_{2}\right) \cap \Delta$ and the origin, respectively. From the geometric description (1) of the normal cone $C$ (see also [N3, Ch. II, Sects. 3 and 4]), it follows that the support of the fibre over the origin of the cone $C$ coincides with the relative tangent cones to $V_{1}$ and $V_{2}$ at $P$. For the definition and properties of relative tangent cones we refer the reader to [ATW, C, K1]. From these papers we can thus deduce the following

Proposition 2. Let $V_{1}, V_{2}$ be two analytic curve germs at the point $P=0 \in \mathbb{C}^{m}$. Then the cone $C_{0}$ is a finite sum of planes; it is the zero cycle iff $V_{1}=V_{2}=V$ and $P$ is a regular point of $V$. Moreover, if one of the germs, say $V_{1}$, is irreducible, all those planes contain the tangent line to $V_{1}$ at $P$.

We are now in a position to present short proofs of two effective formulae (from [CKT, K2]) for the intersection indices of two analytic curves $V_{1}$ and $V_{2}$ in terms of their parametrizations at a point $P$. 
By additivity one may confine oneself to the case where $V_{1}$ and $V_{2}$ are two irreducible curve germs at $P=0 \in \mathbb{C}^{m}$. The problem is evident whenever $V_{1}$ and $V_{2}$ do not have a common tangent line at $P$, as then the intersection index $g$ of $V_{1}$ and $V_{2}$ at $P$ is equal to mult $_{P} V_{1} \cdot \operatorname{mult}_{P} V_{2}$.

Theorem 2. Consider two irreducible curve germs $V_{1}, V_{2}$ at $0 \in \mathbb{C}^{m}$, having a common tangent line, parametrized as follows:

$$
\left(x_{1}=t^{p}, x_{k}=\varphi_{k}(t)\right), \quad\left(x_{1}=t^{q}, x_{k}=\psi_{k}(t)\right), \quad k=2, \ldots, m,
$$

with $\operatorname{ord}_{0} \varphi_{k}>p$ and $\operatorname{ord}_{0} \psi_{k}>q$. Then the generalized intersection index $\widetilde{g}=\left(g_{0}, g_{1}, g_{2}\right)$ of $V_{1}$ and $V_{2}$ at 0 is equal to

$$
\widetilde{g}= \begin{cases}\left(0,0, q^{-1} \sum_{i=1}^{q} \operatorname{ord}_{0}\left(\varphi\left(t^{q}\right)-\psi\left(\varepsilon^{i} t^{p}\right)\right)\right) & \text { if } V_{1} \neq V_{2}, \\ \left(0, p, \sum_{i=1}^{p-1} \operatorname{ord}_{0}\left(\varphi(t)-\varphi\left(\varepsilon^{i} t\right)\right)\right) & \text { if } V_{1}=V_{2}=V,\end{cases}
$$

where $\varepsilon$ is a primitive root of unity of degree $q$ (in the second case $p=q$ and $\varphi(t)=\psi(t))$.

Proof. For the proof, observe that for instance, via the linear change of coordinates $u=x+y, v=x-y$, the normal cone

$$
C:=C_{\left(V_{1} \times V_{2}\right) \cap \Delta}\left(V_{1} \times V_{2}\right)
$$

can be treated as a cone in the product $\mathbb{C}_{x}^{m} \times \mathbb{C}_{y}^{m}$ of the ambient spaces (see e.g. [N3]). In view of Proposition 2, the part $C_{0}$ of $C$ that lies over the origin is a finite union of planes containing the line

$$
L:=\left\{\left(x_{1}, 0, \ldots, 0, y_{1}, 0, \ldots, 0\right) \in \mathbb{C}_{x}^{m} \times \mathbb{C}_{y}^{m}: x_{1}+y_{1}=0\right\} .
$$

Case I: $V_{1} \neq V_{2}$. Then $C=C_{0}$ and the tangent cone $B$ at $0 \in \mathbb{C}^{m}$ to the support of $C$ is a finite union of planes containing $L$.

The generalized intersection index $\widetilde{g}$ is realized by every collection of hyperplanes $\left(H_{1}, H_{2}\right)$ admissible with respect to $B$ (cf. [N4]). Since the hyperplane $\left\{x_{1}-y_{1}=0\right\}$ meets the cone $B$ properly, it can be taken as the first element $H_{1}$ of such an admissible collection of hyperplanes. Proceed now with the intersection algorithm:

Step 0: the result $\varrho_{0}=0$, the remainder $\alpha_{0}=V_{1} \times V_{2}$;

Step 1: the result $\varrho_{1}=0$, the remainder $\alpha_{1}=\alpha_{0} \cdot H_{1}$;

Step 2: for a generic hyperplane $H_{2}$ of the form

$$
\left\{l(x, y):=\sum_{k=2}^{m} a_{k}\left(x_{k}-y_{k}\right)=0\right\},
$$


we have $\alpha_{2}=0$ and $\varrho_{2}=\alpha_{1} \cdot H_{2}=\left(V_{1} \times V_{2}\right) \cdot H_{1} \cdot H_{2}$, and the index $g_{2}$ is the degree at the origin of this 0-cycle. But

$$
\left(x_{1}=t^{p q}, x_{k}=\varphi_{k}\left(t^{q}\right)\right), \quad\left(y_{1}=\tau^{p q}, y_{k}=\psi_{k}\left(\tau^{p}\right)\right), \quad k=2, \ldots, m,
$$

is a parametrization with multiplicity $p q$ of the product $V_{1} \times V_{2}$. Hence and using parametric multiplicity (see e.g. [TW]), we get the required result

$$
\begin{aligned}
g_{2} & =\frac{1}{p q} \operatorname{mult}_{(0,0)}\left(t^{p q}-\tau^{p q}, l\left(\varphi\left(t^{q}\right)-\psi\left(\tau^{p}\right)\right)\right) \\
& =\frac{1}{p q} \sum_{i=1}^{p q} \operatorname{mult}_{(0,0)}\left(\eta^{i} t-\tau, l\left(\varphi\left(t^{q}\right)-\psi\left(\tau^{p}\right)\right)\right) \\
& =\frac{1}{p q} \sum_{i=1}^{p q} \operatorname{ord}_{0}\left(\varphi\left(t^{q}\right)-\psi\left(\left(\eta^{i} t\right)^{p}\right)\right)=\frac{1}{q} \sum_{i=1}^{q} \operatorname{ord}_{0}\left(\varphi\left(t^{q}\right)-\psi\left(\varepsilon^{i} t^{p}\right)\right) ;
\end{aligned}
$$

here $\eta$ is a primitive root of unity of degree $p q$.

CASE II: $V_{1}=V_{2}=V$. Then the normal cone $C$ is the sum of $C_{0}$ and an analytic cone $C^{\prime}$ that lies over $V^{\Delta}=(V \times V) \cap \Delta ; C^{\prime}$ can be parametrized by

$$
\left(t^{p}+p s t^{p-1}, \varphi(t)+s \varphi^{\prime}(t), t^{p}-p s t^{p-1}, \varphi(t)-s \varphi^{\prime}(t)\right) .
$$

The tangent cone $B$ to the support of $C$ at the origin is thus the union of the plane

$$
\left\{\left(x_{1}, 0, \ldots, 0, y_{1}, 0, \ldots, 0\right) \in \mathbb{C}_{x}^{m} \times \mathbb{C}_{y}^{m}: x_{1}, y_{1} \in \mathbb{C}\right\}
$$

and a finite number of planes containing the line $L$ (which form the support of $C_{0}$ ). Therefore, as in Case I, the hyperplane $H_{1}:=\left\{x_{1}-y_{1}=0\right\}$ can be picked up as the first element $H_{1}$ of a collection of hyperplanes which realizes the generalized intersection index. Proceed again with the intersection algorithm:

Step 0: the result $\varrho_{0}=0$, the remainder $\alpha_{0}=V \times V$;

Step 1: $\alpha_{0} \cdot H_{1}=\sum_{i=1}^{p} W_{i}$, where $W_{i}=\left\{\left(t^{p}, \varphi(t), t^{p}, \varphi\left(\varepsilon^{i} t\right)\right)\right\}, i=$ $1, \ldots, p$; the result $\varrho_{1}=W_{p}=V^{\Delta}, g_{1}=p$ and the remainder $\alpha_{1}=\sum_{i=1}^{p-1} W_{i}$;

Step 2: for a generic hyperplane $H_{2}, \alpha_{1} \cdot H_{2}=\varrho_{2}, \alpha_{2}=0$ and the index $g_{2}$ of the result $\varrho_{2}$ at $(P, P)$ is equal to

$$
g_{2}=\sum_{i=1}^{p-1} \operatorname{ord}_{0}\left(\varphi(t)-\varphi\left(\varepsilon^{i} t\right)\right),
$$

and the proof of the theorem is complete.

We immediately obtain the following formulae for the intersection cycles and indices: 
Corollary ([CKT, Th. 1], [K2, Th. 4]). 1) If $V_{1} \neq V_{2}$, then

$$
\begin{aligned}
V_{1} \bullet V_{2} & =i\left(V_{1} \bullet V_{2} ; 0\right) \cdot[0], \\
i\left(V_{1} \bullet V_{2} ; 0\right) & =\frac{1}{q} \sum_{i=1}^{q} \operatorname{ord}_{0}\left(\varphi\left(t^{q}\right)-\psi\left(\varepsilon^{i} t^{p}\right)\right) ;
\end{aligned}
$$

2) if $V_{1}=V_{2}=V$, then

$$
\begin{aligned}
V \bullet V & =1 \cdot[V]+i(V \bullet V ; 0) \cdot[0], \\
i(V \bullet V ; 0) & =\sum_{i=1}^{p-1} \operatorname{ord}_{0}\left(\varphi(t)-\varphi\left(\varepsilon^{i} t\right)\right) .
\end{aligned}
$$

REMARK. By the additivity of the intersection index, formulae (2) and (3) can be applied to calculate $i\left(V_{1} \bullet V_{2} ; P\right)$ for arbitrary analytic curve germs $V_{1}, V_{2}$ at $P$.

\section{References}

[AR] R. Achilles and S. Rams, Intersection numbers, Segre numbers and generalized Samuel multiplicities, Arch. Math. (Basel) 77 (2001), 391-398.

[ATW] R. Achilles, P. Tworzewski and T. Winiarski, On improper isolated intersection in complex analytic geometry, Ann. Polon. Math. 51 (1990), 21-36.

[CKT] J. Chądzyński, T. Krasiński and P. Tworzewski, On the intersection multiplicity of analytic curves in $\mathbb{C}^{n}$, Bull. Polish Acad. Sci. Math. 45 (1997), 163-169.

[C] D. Ciesielska, Relative tangent cone of analytic curves, Ann. Polon. Math. 72 (1999), 191-195.

[D] R. N. Draper, Intersection theory in analytic geometry, Math. Ann. 180 (1969), 175-204.

[CFV] H. Flenner, L. O'Carroll and W. Vogel, Joins and Intersections, Springer, 1999.

[G1] L. J. van Gastel, Excess intersections, Ph.D. thesis, Univ. of Utrecht, 1989.

[G2] - Excess intersections and a correspondence principle, Invent. Math. 103 (1991), 197-221.

[K1] T. Krasiński, The join of algebraic curves, Illinois J. Math., to appear.

[K2] -, Improper intersection of analytic curves, Univ. Iagel. Acta Math. 39 (2001), 153-166.

[N1] K. J. Nowak, Analytic improper intersections I: deformation to the normal cone, Bull. Polish Acad. Sci. Math. 48 (2000), 121-130.

[N2] - Analytic improper intersections II: deformation to an algebraic bicone and applications, ibid., 131-140.

[N3] - Improper intersections in complex analytic geometry, Dissertationes Math. 391 (2001).

[N4] - Remarks on the generalized index of an analytic improper intersection, Ann. Polon. Math. 81 (2003), to appear.

[T] P. Tworzewski, Intersection theory in complex analytic geometry, ibid. 62 (1995), 177-191. 
[TW] P. Tworzewski and T. Winiarski, Cycles of zeroes of holomorphic mappings, Bull. Polish Acad. Sci. Math. 37 (1989), 95-101.

Faculty of Mathematics

University of Łódź

Banacha 22

90-238 Łódź, Poland

E-mail: krasinsk@krysia.uni.lodz.pl
Institute of Mathematics Jagiellonian University Reymonta 4 30-059 Kraków, Poland

E-mail: nowak@im.uj.edu.pl

Reçu par la Rédaction le 5.12.2001

Révisé le 15.5.2002 